Voix et Images

voixetimages

\title{
Le Québec et le far-ouest canadien
}

\section{Anthony Wall}

Volume 28, numéro 1 (82), automne 2002

Noël Audet

URI : https://id.erudit.org/iderudit/000844ar

DOI : https://doi.org/10.7202/000844ar

Aller au sommaire du numéro

\section{Éditeur(s)}

Université du Québec à Montréal

\section{ISSN}

0318-9201 (imprimé)

1705-933X (numérique)

Découvrir la revue

\section{Citer cet article}

Wall, A. (2002). Le Québec et le far-ouest canadien. Voix et Images, 28(1),

182-187. https://doi.org/10.7202/000844ar

Tous droits de reproduction, de rédaction et d'adaptation réservés 2002 C Université du Québec à Montréal
Ce document est protégé par la loi sur le droit d'auteur. L'utilisation des services d'Érudit (y compris la reproduction) est assujettie à sa politique d'utilisation que vous pouvez consulter en ligne.

https://apropos.erudit.org/fr/usagers/politique-dutilisation/ 


\title{
La littérature québécoise hors Québec
}

\section{Le Québec et le far-ouest canadien}

\author{
Anthony Wall, Université de Calgary
}

Géographiquement parlant, les cinq universités ${ }^{1}$ canadiennes dont nous faisons état dans ces lignes sont parmi les plus éloignées possible de Montréal et de Québec. Le relatif isolement provoqué par tant de kilomètres se laisse largement compenser, pourrait-on dire, par la variété des recherches en cours dans divers secteurs des études québécoises et franco-canadiennes. Alors qu'il est évident que nous ne pourrons décrire en détail tout ce qui se fait (ou a été fait) dans les cinq établissements en cause, nous nous proposons de survoler les réalisations, accomplies ou en cours, les plus significatives.

$\mathrm{Au}$ sein de la politique universitaire interne de chaque établissement, les cinq départements dont nous parlons fonctionnent dans des environnements administratifs divers: il s'agit de deux départements «autonomes» de français (Simon Fraser et Victoria), deux départements de langues romanes (Université de Colombie-Britannique et Calgary), un département de langues modernes et d'études culturelles (à Edmonton, où s'enseignent pas moins de onze langues vivantes). Tout cela veut dire que la recherche et l'enseignement dans le grand champ des études québécoises et franco-canadiennes sont diversement affectés par des facteurs tels que la montée spectaculaire des études hispaniques dans l'Ouest canadien, la place des études québécoises dans d'autres programmes universitaires (tels ceux en sciences de l'éducation ou encore dans les programmes d'études canadiennes), la présence d'autres programmes «culturels » (comme les études cinématographiques ou, plus largement, de communication), la collaboration avec les disciplines des sciences sociales, le rôle et la place de la linguistique proprement dite, etc. Certains départements ont l'heureuse possibilité de se lier aux programmes de recherches subventionnés par des Centres ou par des projets concertés ${ }^{2}$, d'autres sont plus «autonomes» de ce point de vue.

Dans ce qui suit, nous donnons un aperçu des activités de recherches selon trois domaines globaux, à savoir: les études littéraires proprement dites, les études de la culture au sens large et les études de la langue et de l'acquisition d'une langue seconde.

\section{Études littéraires}

Ce sont les études littéraires, encore aujourd'hui, qui font la grande force des programmes de recherche et d'enseignement supérieur dans la plupart des départements dont nous faisons état ici. Dans plusieurs cas, les études de la littérature québécoise occupent une place de choix. Le département où se trouve la plus grande concentration de professeurs 
œuvrant dans le champ des études littéraires québécoises est sans doute celui de l'Université de Colombiebritannique à Vancouver. Depuis des années, travaille à Vancouver une équipe importante de chercheurs très actifs en études québécoises, qui effectuent des recherches de pointe dans plusieurs champs: le journal intime, la psychanalyse, Hubert Aquin, l'histoire de la littérature, la littérature comparée (Canada anglais et Québec). De nombreuses publications prestigieuses ont résulté de cette force intellectuelle, parmi lesquelles se trouvent un numéro prisé de la revue Yale French Studies dirigé par Ralph Sarkonak, les études de Réjean Beaudoin sur la naissance de la littérature canadienne, les travaux de Françoise Iqbal et d'André Lamontagne sur Hubert Aquin, les publications de Valérie Raoul sur le journal intime, et bien d'autres.

Cette tradition de qualité se poursuit encore aujourd'hui, avec plusieurs projets dans de nouveaux champs. C'est surtout dans l'étude de l'histoire de la réception que, durant les dernières années, ce département s'est véritablement «spécialisé ». Il s'agit en fait de deux «esthétiques de la réception »: celle, québécoise, de sa propre littérature et de celle du Canada anglais qui se met à lire la littérature québécoise (Beaudoin, Lamontagne, Hodgson, Sarkonak). Cette double activité se poursuit par un nouveau projet en cours sur la réception anglocanadienne de la littérature québécoise (Beaudoin et Lamontagne). Avec l'apport d'Alain-Michel Rocheleau, Vancouver couvre aussi - chose trop rare dans l'Ouest canadien - le domaine essentiel du théâtre (nous y reviendrons plus loin).
On trouve à Calgary autant d'enseignants qui œuvrent dans divers domaines des études québécoises et franco-canadiennes, mais la concentration sur les seules lettres québécoises est nettement moins prononcée qu'à Vancouver, accompagnée qu'elle est d'études sur la culture au sens large, sur la littérature francophone de l'Ouest canadien, et sur la langue française en situation culturelle minoritaire et dans l'enseignement public et post-secondaire. Parmi les recherches en cours sur la littérature québécoise s'inscrivent celles d'Estelle Dansereau sur l'écriture des femmes, celles de Dominique Perron sur la littérature populaire et celles de Claude Romney sur la littérature de jeunesse. Pierre-Yves Mocquais et Anthony Wall ont publié des travaux sur l'œuvre d'Hubert Aquin ${ }^{3}$, le premier poursuivant aujourd'hui des recherches en équipe sur la culture et la langue fransaskoises, le second des études «dialogiques» de l'œuvre aquinienne dans le cadre de l'équipe «Le soi et l'autre» dirigée par Pierre Ouellet à l'Université du Québec à Montréal. L'œuvre de Gabrielle Roy a depuis longtemps attiré l'attention de plusieurs professeurs du département (Glen Campbell, Estelle Dansereau, Claude Romney).

À l'ouest des grands lacs ontariens, Gabrielle Roy est le plus souvent considérée comme la reine de la littérature francophone. C'est certainement le cas à Edmonton, où Paul Dubé poursuit depuis fort longtemps des études de son œuvre, et cela, parallèlement aux autres intérêts qu'il cultive comme, par exemple, ses études dans le domaine, trop peu connu encore, de la littérature francophone dans l'Ouest canadien, sans compter 
son engagement plus que dévoué pour faire reconnaître les droits linguistiques de la minorité francophone dans l'Ouest. Pamela Sing, à la Faculté Saint-Jean (Edmonton), ajoute sa propre énergie intellectuelle à ce même domaine des études de la francophonie de l'Ouest canadien, développant un programme dynamique sur le métissage culturel. Paul Dubé est également rédacteur en chef de la revue Francophonies d'Amérique, qui, depuis plusieurs années déjà, offre aux chercheurs du domaine un lieu public où faire connaître leurs travaux. À Calgary, Glen Campbell travaille aussi dans ce domaine, ayant d'abord fait connaître la poésie de Louis Riel grâce à la première édition complète des œuvres de ce dernier. À l'heure actuelle, il poursuit un projet de recherche sur les écrits littéraires (le plus souvent des poèmes) laissés par les pères Oblats à la fin du XIX ${ }^{\mathrm{e}}$ siècle et au début du $\mathrm{xx}^{\mathrm{e}}$, c'est-à-dire au moment des travaux missionnaires qu'ils ont accomplis surtout en Alberta, mais aussi ailleurs.

Alors que la plupart des départements étudiés ici offrent des cours sur le théâtre contemporain, à la fois sur celui du Québec et celui de plusieurs régions francophones $\mathrm{du}$ Canada (par exemple, l'Acadie ou le Nord de l'Ontario), très peu de collègues, à part Alain-Michel Rocheleau à Vancouver, peuvent se dire «spécialistes » du théâtre. Le travail de Mary Ellen Ross à Victoria sur le théâtre ferronien constitue une heureuse exception par rapport à cette règle générale. Avec les étudiants à Calgary, Dominique Perron a mis sur scène plusieurs pièces classiques du répertoire québécois, conjuguant les études québécoises et les études théâtrales en français.

Le domaine des études québécoises et franco-canadiennes est en train de se transformer dans l'Ouest du Canada. Beaucoup de nouvelles ouvertures se dessinent dans les études comparées: l'Université de l'Alberta offre, depuis de nombreuses années, un très bon programme de littérature comparée où apparaissent souvent des projets de recherches comparatives, dans lesquels les études québécoises et francocanadiennes jouent un rôle important. C'est certainement le cas des recherches en cours que mène Claudine Potvin sur l'écriture des femmes car elle compare, explicitement ou implicitement, l'évolution des enjeux au Québec et en Amérique latine. Il faut dire pourtant que la discipline de «littérature canadienne comparée », qui aurait pour objet l'étude des littératures francophones, anglophones et allophones, n'a pas encore connu de grand essor dans l'Ouest canadien (un programme d'études supérieures a récemment été institué à l'Université de Calgary). Des ressources nouvelles pourraient être exploitées en littérature comparée grâce à une meilleure collaboration entre les chercheurs travaillant dans le champ des études canadiennes «anglophones »et ceux, francophones et francophiles, qui travaillent dans des départements de français, de langues romanes ou de langues modernes. Le travail comparatif de Marie Vautier à l'Université de Victoria constitue un très bon exemple de ce qui, dans l'Ouest du Canada, se propose comme un nouveau type de recherches comparatives; il est possible que de telles études prennent à 
l'avenir une plus grande importance au sein des recherches en littérature.

\section{Études culturelles}

Des enjeux institutionnels plaident en faveur d'une ouverture des chercheurs et des étudiants d'études supérieures aux études québécoises et franco-canadiennes. Les recherches sur la littérature québécoise se décloisonnent et rencontrent d'autres littératures, surtout de langue anglaise; un phénomène concomitant d'ouverture voit les études littéraires se confronter aux études «culturelles». Plusieurs études de grande envergure sont à signaler, trois d'entre elles axées surtout sur la langue et la culture au sens large figurant dans la dernière partie du présent compte rendu.

Mais un mouvement intellectuel ne se fait pas uniquement dans les grandes équipes de recherches; il se donne aussi à voir dans les projets de nouveaux cours et surtout dans le type de recherches qui se dessinent avec une fréquence de plus en plus élevée. Car il est clair que la littérature n'est plus envisagée comme un phénomène isolé, pour ces chercheurs; elle est plutôt appréhendée dans un contexte culturel plus vaste où jouent les autres arts, la politique, la religion, le discours publicitaire, les institutions culturelles telles que les musées et les cinémathèques, etc. C'est dans cette perspective qu'on peut comprendre l'entrée en force des études sur les arts visuels dans les programmes d'enseignement et de recherches. Signalons, à titre d'exemple, l'intérêt d'Estelle Dansereau pour les images culturelles du vieillissement, les études menées par Claudine Potvin à Edmonton sur la muséographie, celles entreprises par le Centre d'études francophones Québec-Pacifique sur les archives de la culture, ou celles du discours publicitaire d'Hydro-Québec poursuivies par Dominique Perron à Calgary.

\section{Études de la langue et acquisition d'une langue seconde}

Dans le domaine des études linguistiques de la langue francocanadienne et québécoise, Douglas Walker (Université de Calgary) étudie en profondeur la variante francoalbertaine. Ce travail se poursuit dans le cadre d'un projet international, dirigé par Jacques Durand (Toulouse), Chantal Lyche (Oslo) et Bernard Laks (Paris X), qui se penche sur l'état actuel du français parlé à travers le monde, en tenant surtout compte des variations phonologiques. Responsable de l'équipe de chercheurs travaillant sur la phonologie canadienne et québécoise, Walker concentre son propre regard sur la région de la Rivière de la Paix en Alberta. Ses recherches entrent dans le champ du Centre de recherches sur la langue de l'Université de Calgary, qui a pour mandat de promouvoir des études sur le langage au sens large, et d'étudier les problèmes d'apprentissage d'une langue en situation minoritaire. À Calgary travaillent dans ce dernier domaine Pierre-Yves Mocquais (sur la langue et la culture fransaskoises) et Sylvie Roy, dont le travail sur le français parlé en milieu minoritaire au Canada concerne non seulement le Centre de recherches sur la langue, mais aussi l'équipe interuniversitaire dirigée par Monica Heller et Normand Labrie du Centre de recherches en éducation franco-ontarienne à 
Toronto. À ces travaux s'ajoutent les recherches linguistiques de John Archibald et de Brian Gill sur l'acquisition d'une deuxième langue, ce dernier collaborant aussi avec Martin Beaudoin de la Faculté Saint-Jean à Edmonton (Gill et Beaudoin organisent le congrès mondial de l'enseignement des langues assisté par ordinateur - «WorldCall» - prévu pour Banff en mai 2003). Il faut mentionner encore les recherches menées au Centre d'études francophones Québec-Pacifique (Université Simon Fraser) et celles de Terry Nadasdi (Université de l'Alberta à Edmonton), qui étudie les variations linguistiques du français à la fois dans le milieu minoritaire et dans l'enseignement par immersion.

$$
\text { *** }
$$

Nous n'avons parlé, très brièvement, que de cinq universités de l'Ouest canadien, les plus importantes des deux provinces occidentales du pays. Elles sont séparées par d'énormes distances géographiques et, malgré toute la bonne volonté du monde, les chercheurs qui y vivent n'ont que trop rarement contact entre eux. À deux exceptions près: le Centre d'études francophones canadiennes de l'Ouest (Saint-Boniface, Manitoba) organise tous les ans, et dans différents établissements de l'Ouest, un colloque international où peuvent participer les chercheurs travaillant dans le champ de l'Ouest francophone; en outre, des chercheurs des départements d'Edmonton et de Calgary se rencontrent tous les ans, lors d'un colloque, pour échanger des communications sur un thème choisi (par exemple, la mémoire culturelle, le mélange des cultures, l'autobiographie). Mais il n'y a tout simplement pas assez d'occasions de rencontres entre Vancouver, Victoria, Edmonton et Calgary. Il en ressort deux conséquences : premièrement, manquent des réseaux forts entre les différentes équipes de chercheurs; deuxièmement, comme les chercheurs se sentent «isolés», un certain phénomène de satellisation s'installe au niveau des recherches (on travaille peut-être trop souvent par rapport à ce qui se fait ailleurs, notamment à Montréal et à Québec, au lieu de développer les possibilités offertes par ce qui se passe intellectuellement ici).

Mais là ne réside pas la toute fin de l'histoire. Il faut aussi composer avec une dure réalité : celle des compressions budgétaires. Il semblerait, dans certains cas, que les départements dits «de langue » soient un peu trop souvent les plus durement touchés par le climat financier de l'enseignement post-secondaire. Le français n'est pas à l'abri de tels courants, et dans cette situation malheureuse se trouvent donc aussi les études québécoises et franco-canadiennes. Face à cette conjoncture, plusieurs transformations, de longue durée, sont à observer. De plus en plus, les inscriptions en français doivent entrer en concurrence avec celles d'autres langues vivantes, notamment l'espagnol. On peut choisir de travailler avec ces autres disciplines ou contre elles. Dans tous les cas, il est sûr que les unités administratives qui enseignent le français doivent se montrer souples vis-à-vis de celles qui «enseignent »d'autres langues. On peut encourager les étudiants à s'intéresser à plus d'une communauté linguistique. L'internationalisation et la 
polyvalence des programmes autant que des recherches constituent pour les études québécoises et françaises d'excellents espoirs de prospérité. Les études québécoises s'ouvrent de plus en plus aux études francophones internationales, non seulement pour se situer par rapport aux études sur d'autres régions francophones $d u$ continent nord-américain, mais aussi pour collaborer aux recherches menées sur la francophonie à l'échelle internationale. Que les recherches avancent de plus en plus sur des bases comparatives (surtout le Québec vis-à-vis du Canada anglophone, des États-Unis et de l'Amérique latine), qu'elles s'aventurent dans des domaines de recherches autres que la littérature (arts visuels, culture populaire, chanson, muséographie, cinéma, discours publicitaire), tout cela constitue, aux yeux de l'auteur de ces lignes, non seulement une bonne tactique pour la survie à long terme, mais avant tout un enrichissement sur le plan intellectuel.

1. Nous parlerons ici des activités de recherche (et parfois d'enseignement) qui s'effectuent aux endroits suivants: l'Université de Victoria (Département de français), l’Université
Simon Fraser à Vancouver (Département de français et Centre d'études francophones Québec-Pacifique), l'Université de Colombiebritannique à Vancouver (Département d'études françaises, hispaniques et italiennes), l'Université de l'Alberta à Edmonton (Department of Modern Languages and Cultural Studies et la Faculté Saint-Jean), l'Université de Calgary (Département d'études françaises, italiennes et espagnoles, le Centre de recherches sur la langue ["Language Research Centre »] de la Faculté des humanités, et le Centre français de la Faculté d'éducation permanente).

2. Les recherches dans les champs des études québécoises et franco-canadiennes à l'Université Simon Fraser profitent évidemment de l'appui et de l'énergie du Centre d'études francophones Québec-Pacifique (dont le mandat comprend la promotion des études sur la langue, la linguistique, la littérature et la culture francophones en Colombiebritannique) ; les recherches analogues à Calgary reçoivent l'appui du Language Research Centre soutenu par la Fondation du Canada pour l'innovation et du Centre français de la Faculté d'éducation permanente (dont le mandat comprend la promotion de l'enseignement et les recherches sur la langue et la culture francophones internationales).

3. Deux autres spécialistes de l'œuvre aquinienne travaillent dans l'Ouest canadien: André Lamontagne (qui nous a donné, il y a dix ans, une étude "intertextuelle » des $œ u v r e s$ romanesques) et Claudine Potvin (qui, en plus de ses recherches sur les écrits contemporains des écrivaines québécoises et latino-américaines, est l'auteure d'une édition critique de la nouvelle «Les rédempteurs ». 